\title{
Analytical evaluation of magnetic pulse deformation of TiNi alloy
}

\author{
E. S. Ostropiko ${ }^{\dagger}$, S. I. Krivosheev, S. G. Magazinov \\ †es-ostropiko@mail.ru
}

Peter the Great St. Petersburg Polytechnic University, St. Petersburg, 195251, Russia

The magnetic pulse method is a method for creating controlled pressure pulses of microsecond duration. Although the method has been known since the 80s, it is usually used for revealing patterns of fracture processes of nonconductive materials. This method was practically not applied to shape memory alloys, with the exception of some works devoted to the three-point bending tests. We adapted the method for high-strain rate uniaxial tension of TiNi alloy specimens. The paper demonstrates the scheme of magnetic pulse method for uniaxial tension. Analytical assessments of the magnitude of the magnetic pressure created by magnetic-pulse drivers under the action of a current pulse, strain in the working part of the sample and the strain rate are carried out. For an analytical solution, the resistance of the material to external forces is proposed to be considered as a piecewise linear approximation to the stress-strain diagram. Analytical solution demonstrates qualitative and quantitative agreement with experiment, even for samples of TiNi alloy with a unique stress-strain diagram. It is a difficult task to estimate the strain rate at short loading pulses of $6-7 \mu \mathrm{s}$. However, in the first approximation, the results show that at the considered loading scheme, the strain rate reaches $6700 \mathrm{~s}^{-1}$ on average and in local areas it is estimated at $10000-12000 \mathrm{~s}^{-1}$. Thus, having the parameters of the material and the parameters of the current pulse, it is possible to estimate the strain of the material with good accuracy and obtain an initial estimate of the strain rate.

Keywords: magnetic pulse method, high strain rate deformation, TiNi alloy.

УДК: $539.3,537.8$

\section{Аналитическая оценка магнитно-импульсного деформирования сплава TiNi}

\author{
Остропико Е. С. ${ }^{\dagger}$, Кривошеев С.И., Магазинов С. Г. \\ Санкт-Петербургский Политехнический Университет Петра Великого, Санкт-Петербург, 195251, Россия
}

\begin{abstract}
Метод магнитно-импульсного нагружения - это метод создания коротких импульсов давления, длительностью в несколько микросекунд. Хотя метод известен еще с 80-х годов, как правило, его применяют для определения закономерностей при разрушении непроводящих материалов. К сплавам с памятью формы подобные схемы практически не применялись, за исключением отдельных работ, посвященных испытанию проволочных образцов на трехточечный изгиб. Мы адаптировали метод, который используется для исследования механики разрушения материалов, для одноосного деформирования на примере образцов из сплава TiNi. B работе продемонстрирована схема магнитно-импульсного нагружения для одноосного растяжения, проведена аналитическая оценка величины магнитного давления, создаваемого магнитно-импульсными драйверами под действием импульса тока, деформации в рабочей части образца и скорости деформирования. Для возможности аналитического решения сопротивление материала внешним усилиям предлагается рассматривать как кусочно-линейное приближение к диаграмме деформирования. Расчет деформации показал качественное и количественное соответствие с экспериментом, даже на образцах из сплава TiNi с весьма характерной диаграммой деформирования. Экспериментально оценить скорость на коротких импульсах нагружения в 6-7 мкс - задача нетривиальная. Тем не менее, в первом приближения аналитическая оценка показывает, что при рассмотренной схеме нагружения процесс деформирования в среднем развивает скорость до $6700 \mathrm{c}^{-1}$, а на отдельных участках оценивается в 10000-12000 $\mathrm{c}^{-1}$. Таким образом, имея в распоряжении только параметры материала и параметры импульса тока можно аналитически с неплохой точностью оценить величину деформации материала и получить первичную оценку скорости деформирования.
\end{abstract}

Ключевые слова: магнитно-импульсный метод, высокоскоростное деформирование, сплав TiNi. 


\section{1. Введение}

Использование энергии электромагнитных полей открыло уникальные перспективы для технологий обработки, исследования материалов, решения различных технологических задач: магнитноимпульсная формовка, электромагнитное сжатие, штамповки листового металла, магнитноимпульсная сварка и др. [1-5]. Еще в 1958 году в Женеве на выставке мирного использования ядерной энергии «General Dynamics Corp» продемонстрировала первое магнитно-импульсное оборудование для обработки трубчатых объектов [5].

Целый класс способов применения энергии магнитных полей - нагружение материалов импульсами магнитного давления длительностью в несколько микросекунд, магнитно-импульсное нагружение [1-10]. Одним из методов является магнитно-импульсное нагружение образцов с макродефектом типа трещин, важная особенность которого в том, что деформирование материала происходит без предварительного формирования в нем напряженного состояния, что может быть важно при испытании материалов с пространственной анизотропией [11-14]. Несмотря на то, что методика известна еще с 80-х годов [8], ее вариации зачастую применяют именно для определения закономерностей при разрушении различных материалов, в том числе проводящих [10,15]. Можно отметить работу [16], где показаны эксперименты по электромагнитному растяжению образцов из магниевого сплава, но там предлагается схема, которая использует токи, наведенные в самом образце.

Методы магнитно-импульсного нагружения известны на протяжении десятилетий, но к сплавам с памятью формы подобные схемы практически не применялись, за исключением отдельных работ. Например, в [17] авторы показали вариацию магнитно-импульсной установку для испытания проволочных образцов на трехточечный изгиб.

В целом очевидно, что этот подход может быть использован для исследования функциональных и механических свойств материалов, связанных с изменением формы или деформации при различных скоростях деформирования, а не только для исследования свойств катастрофического перехода, вызванного разрушением. Ограничения на скорость деформации связаны с возможностями генераторов импульсного тока и магнитных систем. В этом исследовании мы попытались адаптировать метод, который обычно используется для исследования разрушения материалов, для реализации высокоскоростного одноосного растяжения на примере сплава TiNi с памятью формы.

Таким образом, цель работы - адаптировать метод, который обычно используется для исследования механики разрушения материалов, для деформирования в режиме одноосного растяжения, продемонстрировать новую схему магнитно-импульсного нагружения для одноосного растяжения, и провести аналитическую оценку деформации и скорости деформирования на примере сплава TiNi.

\section{2. Экспериментальная установка}

На Рис. 1 схема экспериментальной установки, которая состоит из генератора импульсного тока (ГИТ) и магнитно-импульсных драйверов (МИД), которые представляют из себя плоские медные шины. $C, L, R$ - емкость, индуктивность и сопротивление генератора, а $S-$ разрядное устройство. Генератор создает ток $I$ за счет разряда конденсаторной батареи $C$. В классической схеме для исследования свойств разрушения ток протекает через МИД, вставленный в разрез образца с макродефектом типа трещины $[1,6]$. Когда через петлю протекает ток, он создает магнитное поле, которое взаимодействует с током в параллельном участке МИД. Возникает магнитное давление, которое приводит к деформированию и разрушению образца. В нашей схеме, адаптированной для одноосного растяжения, ГИТ остается тем же, но протекающий ток распараллеливается на два МИД, вставленных симметрично в разрезы специально подготовленного образца, как показано на Рис. 1. Пространство между драйверами и образцом, как и пространство внутри петель заполняется диэлектриком, так что образец «надевается» довольно плотно. Форма образца подобрана таким образом, чтобы деформация накапливалась именно в рабочей части: толщина сечения рабочей части (отмечена желтым на Рис. 1) тоньше, чем толщина оставшейся плоскости образца. За счет этой разницы плоскости приложения магнитного давления смещаются практически плоскопараллельно, в то время, как рабочая зона деформируется в режиме растяжения. Симметрия в форме образца и в приложении сил позволяет дополнительно не фиксировать образец, достаточно лишь аккуратно его установить на драйверы. После деформирования измеряли остаточную деформацию образцов.

Общий импульс тока измеряется осциллографом при помощи пояса Роговского. Параметры импульса тока можно варьировать, меняя заряд конденсатора и индуктивность ГИТ. В рассматриваемом случае он представляет из себя затухающую синусоиду с отношением соседних амплитуд 0.6 и периодом 6-7 мкс

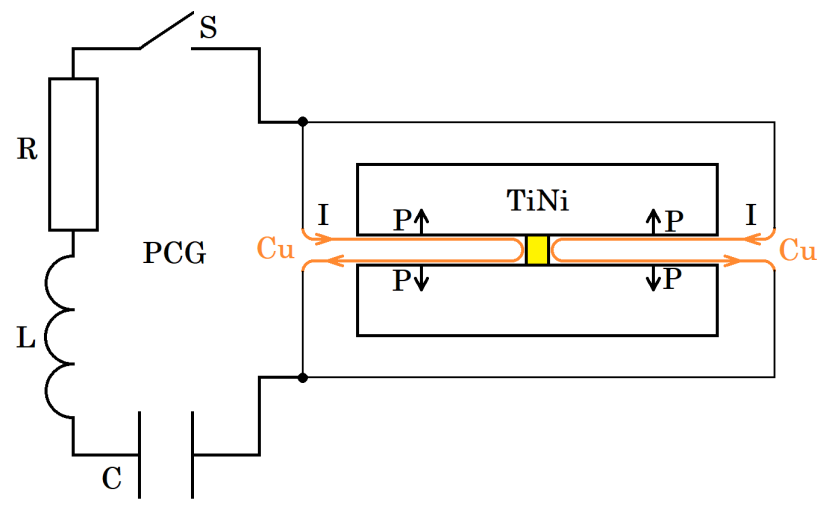

Pис. 1. (Color online) Схема магнитно-импульсной установки на растяжение с образцом из сплава TiNi. Рабочая часть отмечена желтым.

Fig. 1. (Color online) Schematic of a tensile magnetic-pulse setup with a TiNi alloy specimen. Working part colored yellow. 
(Рис. 2). Образцы были изготовлены с помощью электроэрозионной резки из пластины толщиной 2 мм. Сечение рабочей части $-1 \times 1$ мм, высота -2.5 мм.

\section{3. Аналитическая оценка}

\section{1. Магнитное давление}

Для аналитической оценки в первую очередь надо определить величину магнитного давления в проводниках.

На Рис. 3 представлена схема поперечного сечения магнитно-импульсного драйвера шириной $c$ и толщиной $d$.

Геометрически видно, что магнитное давление в случайной точке нижней плоскости драйвера формируется со стороны двух участков (I, II) верхней плоскости драйвера. Для определения магнитного давления необходима горизонтальная составляющая вектора напряженности магнитного поля $H_{x}$. Если предположить равномерное распределения тока в сечении драйвера, то напряженность магнитного поля от точечного участка проводника с током можно определить по формуле:

$$
\begin{gathered}
H=\frac{j d S}{2 \pi l} ; \\
H_{x}=\cos \alpha \frac{j d S}{2 \pi l}=\frac{j d S \Delta h}{2 \pi\left(\Delta h^{2}+b^{2}\right)},
\end{gathered}
$$

где $j=I / c d-$ плотность тока, при условии равномерного распределения.

Напряженность магнитного поля в точке $H_{x}^{\mathrm{II}}$, со стороны всего участка II можно определить, проинтегрировав горизонтальную составляющую вектора магнитного поля по толщине драйвера $d$ и по ширине участка II $a$ :

$$
\begin{aligned}
H_{x}^{\mathrm{II}} & =\int_{h}^{h+d a} \int_{0}^{j} \frac{j \Delta h}{2 \pi\left(\Delta h^{2}+b^{2}\right)} d b d \Delta h= \\
& =\frac{j}{4 \pi}\left(a \ln \left(\frac{a^{2}+(h+d)^{2}}{h^{2}+a^{2}}\right)+\right. \\
& \left.+2(h+d) \tan ^{-1}\left(\frac{a}{h+d}\right)-2 h \tan ^{-1}\left(\frac{a}{h}\right)\right),
\end{aligned}
$$

где $h$ - расстояние между плоскостями драйвера.

Чтобы получить магнитное давление $P_{m}^{\text {II }}$ со стороны участка II не в точке, но в сечении нижней плоскости драйвера необходимо силу Лоренца проинтегрировать по сечению нижней плоскости проводника $d$ :

$$
\begin{aligned}
P_{m}^{I I} & =\int_{h}^{h+d} \mu j H_{x}^{\mathrm{II}} d h= \\
& =\mu \frac{j^{2}}{4 \pi}\left((a h+2 a d) \ln \left(\frac{a^{2}+\left(h^{2}+2 d^{2}\right)}{a^{2}+(h+d)^{2}}\right)-a h \ln \left(\frac{a^{2}+(h+d)^{2}}{a^{2}+h^{2}}\right)+\right. \\
& +\left(a^{2}-h^{2}-2 d^{2}-4 d h\right) \tan ^{-1}\left(\frac{a d}{\left.a^{2}+h^{2}+2 d^{2}+3 d h\right)}\right)- \\
& \left.-\left(a^{2}-h^{2}\right) \tan ^{-1}\left(\frac{a d}{\left.a^{2}+h^{2}+d h\right)}\right)+2 d^{2} \tan ^{-1}\left(\frac{a}{h+2 d}\right)\right),
\end{aligned}
$$

где $\mu$-магнитная проницаемость.

Давление $P_{m}^{\mathrm{I}}$ со стороны участка I определяется аналогичной формулой. Таким образом, суммируя значения

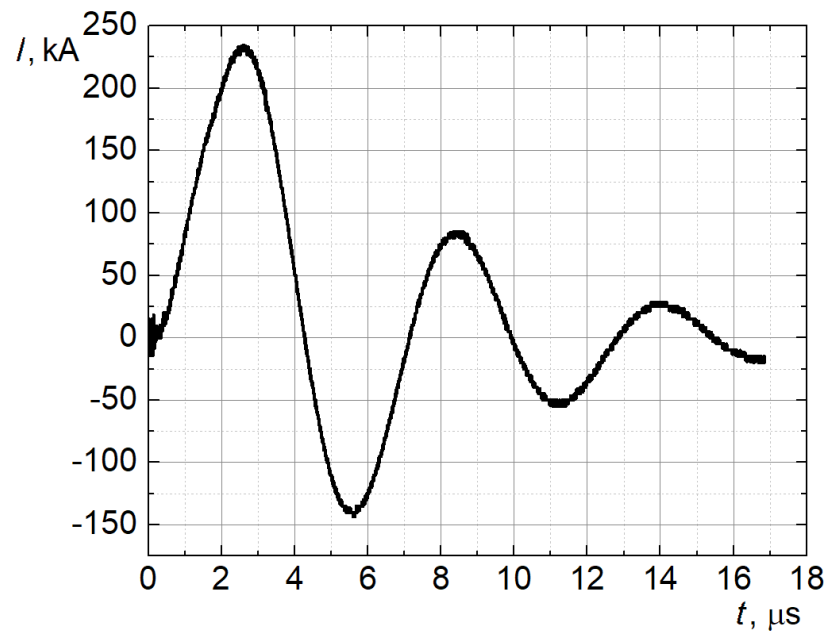

Рис. 2. Осциллограмма импульса тока.

Fig. 2. Discharge current waveform.

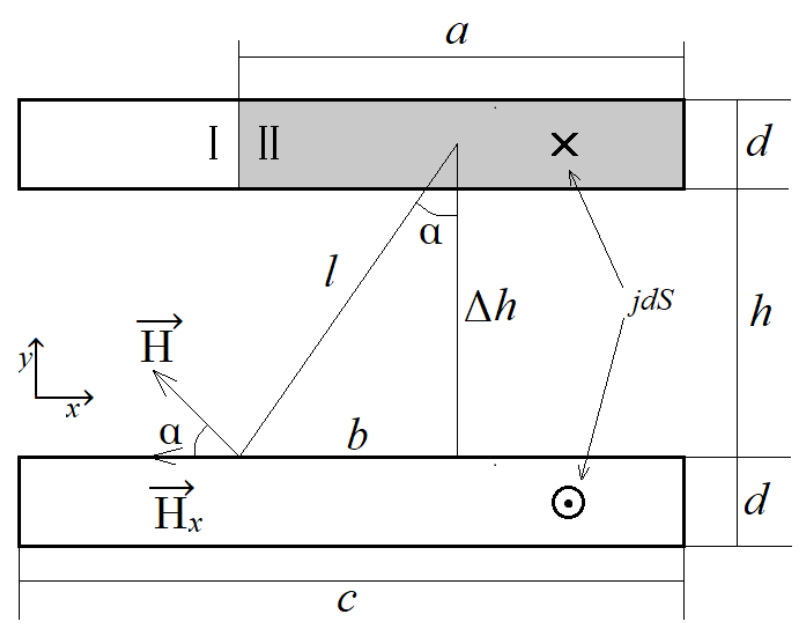

Рис. 3. Схема поперечного сечения магнитно-импульсного драйвера.

Fig. 3. Schematic cross-section of a magnetic pulse driver.

$P_{m}^{\text {I }}$ и $P_{m}^{\text {II }}$ получим величину магнитного давления в каждом сечении нижней плоскости МИД со стороны верхней плоскости МИД. То есть, при условии равномерного распределения тока, можно получить распределение магнитного давления по всей ширине нижнего участка МИД и определить его среднее значение $P_{m}^{a v}$.

Известно, что при высокочастотных и переходных процессах ток распределяется неравномерно. Это можно учесть, введя дополнительный коэффициент $k_{f}$. Тогда при неравномерном распределении, с учетом поправки, магнитное давление выглядит следующим образом:

$$
P_{M}=k_{f} P_{m}^{a v}
$$

При условии $c \gg h$ можно положить $k_{f}=1$, в других случаях $0<k_{f}<1$, и его можно определить на основе численного расчета для тока рассматриваемой частоты в COMSOL Multiphysics. Аналогичная ситуация рассматривалась в $[6,9]$. В нашем случае, на основе численного расчета для тока рассматриваемой частоты $k_{f} \approx 0.83$. 


\section{2. Уравнение движения}

В силу симметрии образца и приложенных сил (Рис. 4), уравнение движения можно описать следующим образом:

$$
m \ddot{y}(t)=F(t)-S_{0} \sigma(t),
$$

где $m$ - массы половины образца (в силу симметрии), $S_{0}$ - площадь сечения рабочей части, $\sigma(t)-$ напряжение в рабочей части образца при растяжении.

Действующая сила $F(t)$ определяется через магнитное давление $P_{M}$, а магнитное давление напрямую связано с импульсом тока, форма которого представляет из себя затухающую синусоиду (Рис. 2). Осцилограмма принимает отрицательные значения, в зависимости от направления тока, но для магнитного давления направление тока роли не играет. Поэтому давление выражается через квадрат синуса, а экспонента в знаменателе характеризует затухание:

$$
F(t)=S P_{M}(t)=S P \frac{\sin ^{2}(2 \pi f t)}{e^{2 t / \tau}},
$$

где $f$ - частота импульса тока, $\tau-$ постоянная времени затухания, $S$ - площадь поверхности нагружения. Параметр $P$ подбирается так, чтобы амплитудное значение $P_{M}$, рассчитанное по формуле (4) совпадало с амплитудным значением $P_{M}(t)$, рассчитанным по формуле (6).

Для возможности аналитического решения уравнения (5) сопротивление материала можно представить в кусочно-линейном приближении как уравнение прямой:

$$
\sigma(t)=a \varepsilon(t)+b=a \frac{y(t)}{l_{0}}+b,
$$

где $l_{0}-$ начальная длина рабочей части образца; $a, b-$ параметры прямой на кусочно-линейных участках, которые легко определяются по границам этих участков (Рис. 5). В скобках на рисунке показаны границы отрезков кусочно-линейного приближения. Диаграмма деформирования, конечно, получена квазистатически и по-хорошему требует учета влияния скорости деформирования на пределы переориентации и текучести, однако для первого аналитического приближения решено было использовать ее.

Подставляя уравнения (6), (7) в (5), и сделав небольшие преобразования получим уравнение движения образца под действием магнитно-импульсного давления.

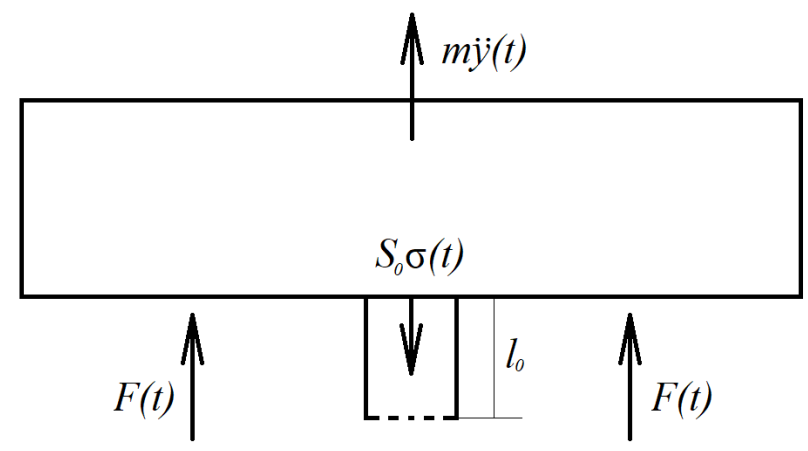

Рис. 4. Схема задачи.

Fig. 4. Schematic statement of task.

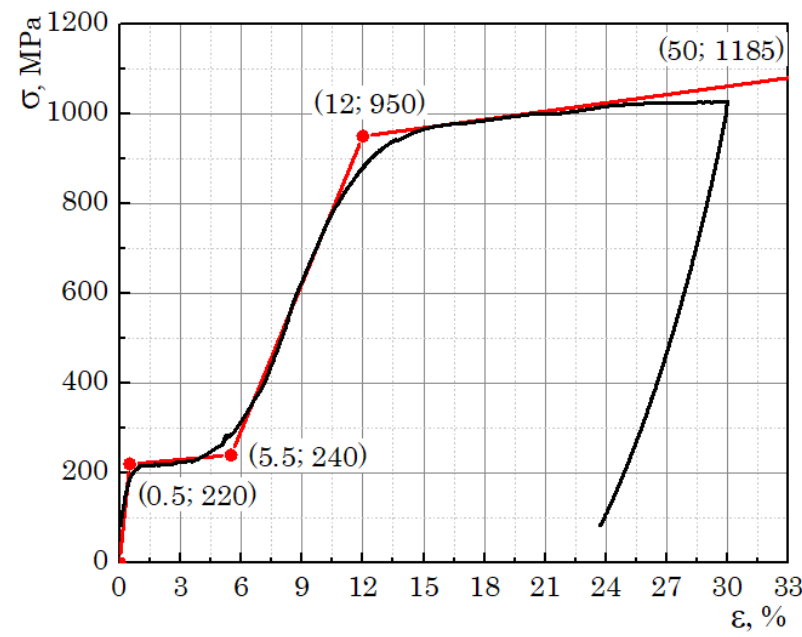

Pис. 5. (Color online) Кривая деформирования сплава TiNi и еe кусочно-линейное приближение. В скобках показаны границы отрезков кусочно-линейного приближения.

Fig. 5. (Color online) TiNi stress-strain curve and its piecewise linear approximation. The coordinates of the vertices of the segments of the piecewise linear approximation are in brackets.

Учитывая, что в начальный момент времени материал находится в покое, получим задачу Коши:

$$
\left\{\begin{array}{c}
\ddot{y}(t)+A y(t)=-B+\frac{F}{2} e^{-\frac{2 t}{\tau}}-\frac{F}{2} e^{-\frac{2 t}{\tau}} \cos 4 \pi f t \\
\dot{y}(0)=0 \\
y(0)=0
\end{array},\right.
$$

где $A=S_{0} a / m l_{0}, B=S_{0} b / m, F=S P / m$.

Задачу можно решить не только численными методом, но и аналитически. Общее решение в аналитическом виде выглядит следующим образом:

$$
\begin{aligned}
y(t) & =C_{1} \sin \sqrt{A} t+C_{2} \cos \sqrt{A} t-\frac{B}{A}+\frac{F \tau^{2}}{2 A \tau^{2}+8} e^{-\frac{2 t}{\tau}}+ \\
& +\frac{8 F \pi f \tau^{3}}{\left(4+A \tau^{2}-16 \pi^{2} f^{2} \tau^{2}\right)^{2}+256 \pi^{2} f^{2} \tau^{2}} e^{-\frac{2 t}{\tau}} \sin 4 \pi f t+ \\
& +\frac{8 F \pi^{2} f^{2} \tau^{2}-2 F \tau^{2}-\frac{F A \tau^{4}}{2}}{\left(4+A \tau^{2}-16 \pi^{2} f^{2} \tau^{2}\right)^{2}+256 \pi^{2} f^{2} \tau^{2}} e^{-\frac{2 t}{\tau}} \cos 4 \pi f t .
\end{aligned}
$$

Остается определить коэффициенты $C_{1}, C_{2}$ из задачи Коши на каждом участке кусочно-линейного приближения кривой деформирования, сложить приращения $y(t)$ на каждом линейном участке в общую последовательность.

\section{4. Результаты и сравнение с экспериментом}

Параметры для расчета амплитуды магнитного давления. Размеры МИД: $d=0.3 \mathrm{мм}, h=1.3 \mathrm{мм}, c=4$ мм. Магнитная постоянная $\mu=4 \pi \cdot 10^{-7}$. Амплитудные значения тока варьировались и составили 95, 100, 108, 118,123 кА в одном магнитно-импульсном драйвере. 
С помощью уравнения (6) получили амплитуду магнитного давления: 148, 165, 190, 227, 248 МПа, соответственно.

Параметры для уравнения движения. Параметры образца: $m=2.5$ г, площадь сечения рабочей части $S_{0}=1 \mathrm{Mм}^{2}$, площадь поверхности нагружения $S=72.5 \mathrm{Mm}^{2}$, начальная длина рабочей части $l_{0}=1.25$ мм, параметры кусочно-линейных участков $a$ и $b$ определяются по вершинам этих участков (Рис. 5). Частота импульса тока $f=143000$, постоянная затухания $\tau \approx 6.5 \cdot 10^{-6}$.

Далее, используя формулу (9), собрав все приращения в общую последовательность, получим кривую зависимости деформации $\varepsilon(t)=y(t) / l_{0}$ от времени (Рис. 6).

На Рис. 7 а показана зависимость расчетного максимума деформации и остаточной деформации, полученной в эксперименте, от приложенной нагрузки. Характер зависимостей и порядок значений совпадает.

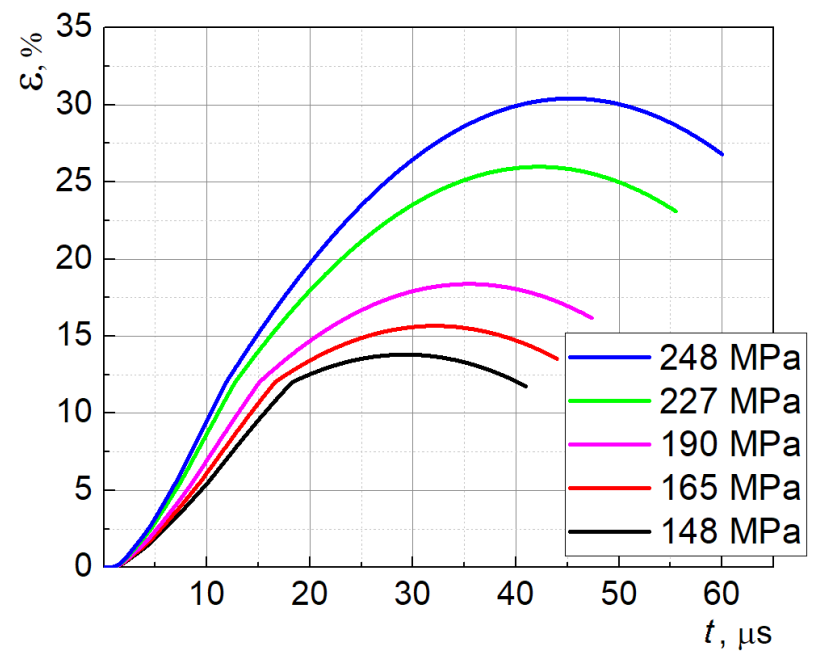

Рис. 6. (Color online) Кривые зависимости деформации от времени при различных магнитных давлениях.

Fig. 6. (Color online) Strain versus time curves at various magnetic pressure.

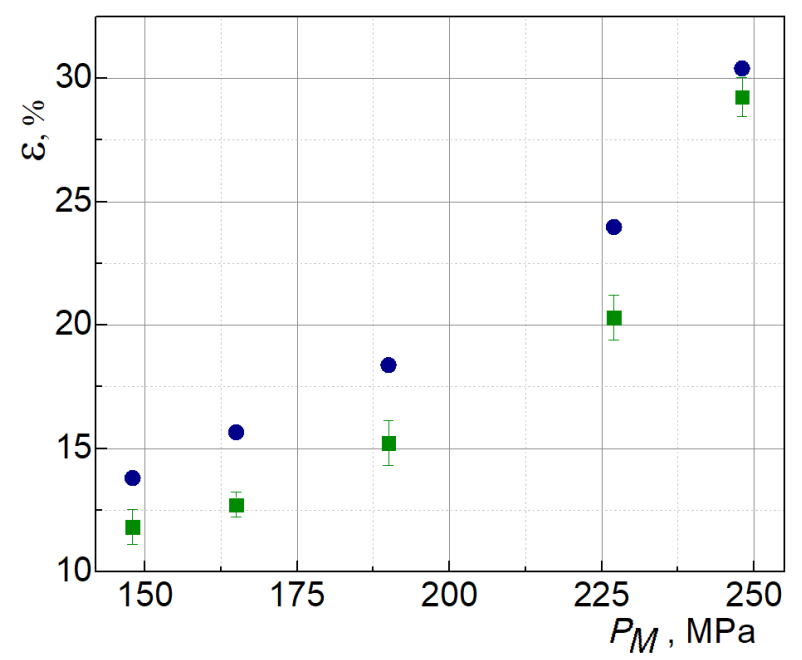

Разница лишь в том, что аналитика не учитывает упругий возврат деформации при снятии нагрузки, но если предположить, что возвращается порядка 2 - 3\% упругой деформации, что недалеко от истины, то можно получить очень близкие значения экспериментальных и расчетных величин остаточной деформации.

На Рис. 7b показана зависимость скорости деформирования от амплитуды магнитного давления. Скорость определялась из Рис. 6 как отношение максимального значения деформации к соответствующему моменту времени. Оценочная скорость деформирования в зависимости от нагрузки и степени деформации находится в диапазоне $4700-6700 c^{-1}$.

\section{5. Заключение}

В работе продемонстрирована схема магнитно-импульсного нагружения для деформирования в режиме одноосного растяжения на примере образцов из никелида титана. Предложена аналитическая оценка величины магнитного давления, деформации и скорости деформирования.

Расчет деформации показал достаточно хорошее качественное и количественное соответствие с экспериментом, даже на образцах из сплава TiNi с весьма характерной диаграммой деформирования. Более того, аппроксимировать кривую деформирования кусочнолинейными участками можно с более высокой точностью, с учетом влияния скорости на пределы переориентации и текучести, что приведет к более точной оценке деформации. И, конечно, нет привязки к конкретному материалу.

Экспериментально оценить скорость на коротких импульсах нагружения в 6-7 мкс - задача нетривиальная. Аналитическая оценка требует экспериментального подтверждения, что, несомненно, будет сделано впоследствии, поскольку она не учитывает волновые процессы, которые развиваются в материале под динамической

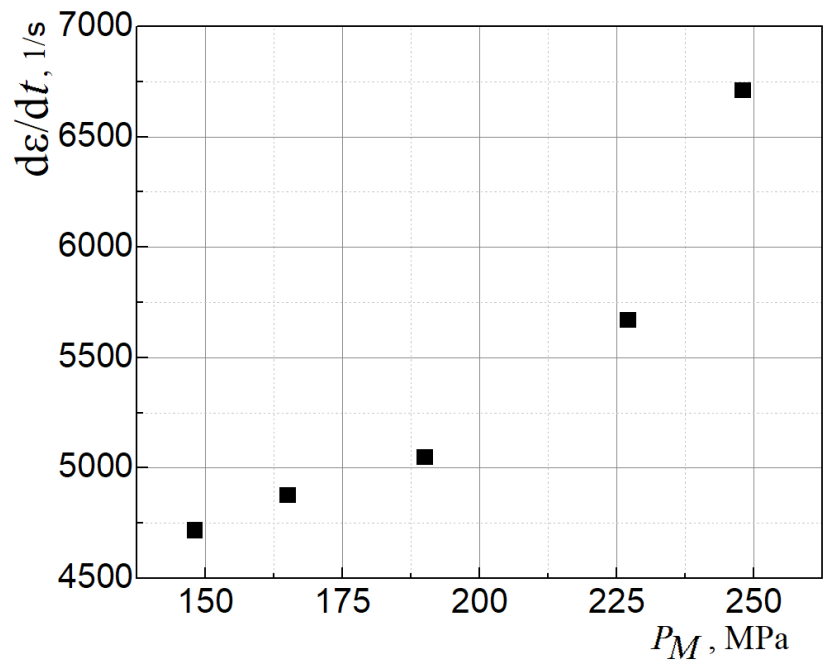

$\mathrm{b}$

Pис. 7. (Color online) Зависимость расчетной деформации (•) и экспериментальной остаточной деформации (घ) от амплитуды магнитного давления (a), оценка скорости накопления деформации в зависимости от амплитуды магнитного давления (b).

Fig. 7. (Color online) Dependence of the calculated strain $(\bullet)$ and experimental residual strain ( $\square$ ) on the magnetic pressure (a), estimation of the strain rate $(b)$. 
нагрузкой. Тем не менее, в первом приближения аналитическая оценка показывает, что при рассмотренной схеме нагружения процесс деформирования в среднем развивает скорость до $6700 \mathrm{c}^{-1}$, а на отдельных участках оценивается в $10000-12000 \mathrm{c}^{-1}$.

Таким образом, имея в распоряжении только параметры материала и параметры импульса тока можно аналитически с неплохой точностью оценить величину деформации материала и получить первичную оценку скорости деформирования.

Благодарности / Acknowledgements. Исследование выполнено при финансовой поддержке РФФИ в рамках научного проекта № 19-32-60035. Экспериментальная реализация магнитно-импульсного нагружения выполнена при поддержке проекта 5-100 на базе СанктПетербургского Политехнического Университета Петра Великого. / The reported study was funded by RFBR, project number 19-32-60035. The experiments on magnetic-pulse loading were carried out with the support of the Academic Excellence Project 5-100 proposed by Peter the Great St. Petersburg Polytechnic University.

\section{Литература/References}

1. V. Psyk, D. Risch, B. L. Kinsey, A. E. Tekkaya, M. Kleiner, J. Mater. Process. Technol. 211 (5), 787 (2011). Crossref

2. V.A. Glushchenkov, V.F. Karpukhin. Technology of Magnetic-Impulse Materials Processing. Samara, Publishing House "Fedorov" (2014) 193 p. (in Russian) [В.А. Глущенков, В.Ф. Карпухин. Технология магнитно-импульсной обработки материалов. Самара, издательский дом «Федоров» (2014) 193 с.]
3. V.A. Glushchenkov. Key Eng. Mater. 684, 511 (2016). Crossref

4. T. Aizawa, M. Kashani, K. Okagawa. Welding Journal. 86, 119 (2007).

5. Yu. V. Batygin, E. A. Chaplygin, O.S. Sabokar. Elektroteh. elektromeh. 5, 35 (2016). $\underline{\text { Crossref }}$

6. S. Krivosheev, S. Magazinov, D. Alekseev. MATEC Web Conf. 145, 05006 (2018). Crossref

7. N.V. Korovkin, S.I. Krivosheev, S.G. Magazinov, V. K. Slastenko. Int. J. Mech. 9, 293 (2015).

8. K. R. Chandar, W. G. Knauss. Int. J. Fract. 20, 209 (1982). $\underline{\text { Crossref }}$

9. J.R. Asay, T. Ao, T.J. Vogler, J.-P. Davis, G. T. Gray III, J. Appl. Phys. 106, 073515 (2009). Crossref

10. S.G. Magazinov, S.I. Krivosheev, Yu.E. Adamyan, D. I. Alekseev, V.V. Titkov, L.V. Chernenkaya. Mater. Phys. Mech. 40, 117 (2018). Crossref

11. G.I. Kanel, S. V. Razorenov, V.E. Fortov. Joint 20th AIRAPT - 43th EHPRG. Karlsruhe, Germany (2005) 119921.

12. G. I. Kanel, S. V. Razorenov, G. V. Garkushin, A. S. Savinykh. J. Phys. Conf. Ser. 946, 012039 (2018). Crossref

13. Y. Meshcheryakov, A. Divakov, N. Zhigacheva, G. Konovalov. Proc. Struct. Int. 2, 477 (2016). Crossref

14. G. G. Savenkov, Yu. I. Meshcheryakov, B. K. Barakhtin, N. V. Lebedeva. J. Appl. Mech. Tech. Phys. 55, 896 (2014). $\underline{\text { Crossref }}$

15. V. A. Morozov, Yu. V. Petrov, V.D. Sukhov. Tech. Phys. 64 (5), 642 (2019). Crossref

16. J. R. Xu, H.P. Yu, C. F. Li. J. Mater.Eng.Perform. 22 (4), 1179 (2013). Crossref

17. A. Gruzdkov, S. Krivosheev, Yu. Petrov, A. Razov, A. Utkin. Mater. Sci.Eng. A. 481-482, 105 (2008). Crossref 